\title{
Tai Chi for Chronic Obstructive Pulmonary Disease (COPD): An Overview of Systematic Reviews
}

\author{
Luping Yang $\mathbb{B}^{1, *}$ \\ Dongling Zhong ${ }^{2, *}$ \\ Yue Zhang ${ }^{2}$ \\ Yuxi $\mathrm{Li}^{2}$ \\ Tianyu Liu $^{3}$ \\ Yaling Zheng ${ }^{2}$ \\ Wei Wang ${ }^{4}$ \\ Juan $\mathrm{Li}\left(\mathbb{D D}^{2}\right.$ \\ Li Guan ${ }^{5}$ \\ Rongjiang Jin ${ }^{2}$ \\ 'Department of Rehabilitation \\ Technology, Sichuan Nursing Vocational \\ College, Chengdu, 6I 0037, People's \\ Republic of China; ${ }^{2}$ Department of \\ Rehabilitation, Chengdu University of \\ Traditional Chinese Medicine, Chengdu, \\ 610037, People's Republic of China; \\ ${ }^{3}$ Department of Sport, Chengdu \\ University of Traditional Chinese \\ Medicine, Chengdu, 610037, People's \\ Republic of China; ${ }^{4}$ Department of \\ Rehabilitation, Kunming Municipal \\ Hospital of Traditional Chinese Medicine, \\ Kunming, 650000, People's Republic of \\ China; ${ }^{5}$ Department of Rehabilitation, \\ People's Hospital of Fushun County, \\ Zigong, 643000, People's Republic of \\ China
}

*These authors contributed equally to this work

\begin{abstract}
Objective: Since current systematic reviews (SRs) show that results of effectiveness on Tai Chi for chronic obstructive pulmonary disease (COPD) are inconsistent, the purpose of this study is to find the reasons of the disparity by comprehensively appraising the related SRs. Methods: Six databases were systematically searched from the inception date to April 17, 2021. The methodological quality, the risk of bias, the reporting quality, and the quality of evidence were independently assessed by two reviewers with the AMSTAR 2, ROBIS, PRISMA, and GRADE.

Results: A total of 12 studies met the inclusion criteria: 10 SRs were rated critically low quality and two SRs were low quality by AMSTAR 2. By the ROBIS, four out of 12 SRs were rated as "low risk". According to PRISMA, nine out of 12 SRs were adequately reported over $80 \%$. With the GRADE tool, three out of 12 SRs rated the FEV1 as "Moderate", one out of 12 SRs $(1 / 12,9 \%)$ rated the FEV1/FVC (\%) as "Moderate", three out of 12 SRs assessed the 6MWD as "Moderate", three out of 12 SRs evaluated the SGRQ as "Moderate", and the remaining evidence was fully rated as "Low" or "Very Low".

Conclusion: We found that the methodological quality, risk of bias, reporting quality, and quality of evidence of the included SRs on Tai Chi for COPD were suboptimal. These limitations may have a negative impact on SRs, consequently leading to inconsistent results. Further wellconducted SRs with less risk of bias, more rigorous methodology, normative reporting and highquality of evidence are needed to provide robust evidence on Tai Chi for COPD.
\end{abstract}

Registration Number: This study has been registered in the PROSPERO International Prospective Register of Systematic Reviews (registration number: CRD42019126600).

Keywords: Tai Chi, COPD, AMSTAR 2, ROBIS, PRISMA, GRADE, overview

\section{Background}

Chronic obstructive pulmonary disease (COPD) is a common, preventable and treatable disease that is characterized by persistent respiratory symptoms and airflow limitation due to airway and/or alveolar abnormalities. ${ }^{1}$ Globally, there are around 3 million deaths annually caused by COPD. ${ }^{2}$ By $2030,{ }^{3}$ more than 4.5 million people may die from COPD and related diseases every year. Currently, pharmacological therapies are considered as the first-line treatment for COPD. However, medications mainly focus on relieving symptoms rather than improving pulmonary function and quality-of-life. ${ }^{4}$ Guidelines ${ }^{5}$ for COPD indicate that exercise, as the core content of pulmonary rehabilitation, should be added to routine treatments for patients with moderate-to-severe COPD.

Tai Chi, as a traditional Chinese exercise with unique cultural characteristics, has been widely used for the rehabilitation in COPD. Clinical studies have
Rongjiang Jin

Email cdzyydxjrj@I26.com 
manifested that Tai Chi was beneficial for COPD, due to its effectiveness of strengthening and stretching the body, improving the flow of blood and other fluids throughout the body, and improving balance. ${ }^{6}$ With the development of evidence-based medicine, systematic reviews (SRs) have become the standard approach for clinicians to make decisions. $^{7-9}$ However, evidence of the effectiveness on Tai Chi for COPD is inconsistent. For example, $\mathrm{Wu}$ et $\mathrm{al}^{10}$ found that Tai Chi significantly increased the forced expired volume in one second per predicted (FEV1\%), but Ngai et al $^{11}$ showed negative results. In such circumstances, the clinicians and policy-makers may be inundated with inconsistent evidence.

The disparity mainly comes from the methodology, risk of bias, reporting, and the quality of evidence of outcomes. Low methodologic quality may cause the results of SRs to be less reliable. ${ }^{12}$ Bias in the design or progress of SRs may lead to uncertain conclusions. Reporting quality is crucial for both authors and readers ${ }^{13}$ to obtain the information, yet a large number of suboptimal reporting persists. ${ }^{14}$ Because of the inadequate reporting and low quality of evidence, readers cannot fully assess the strengths and weaknesses of SRs, which ultimately means that the findings of SRs cannot be implemented into clinical practice. ${ }^{15,16}$ Therefore, a comprehensive overview of SRs, which can comply data from multiple SRs ${ }^{17}$ and reduce disparity between SRs, is more intuitive than numbers of SRs with low quality and inconsistent recommendations.

To date, no overview of related SRs on Tai Chi for COPD has been conducted. Our study aimed to conduct a comprehensive overview and to critically appraise the methodology, risk of bias, reporting quality, and quality of evidence of these SRs, by using the tools of A Measurement Tool to Assess Systematic Reviews 2 (AMSTAR 2), Risk of bias in systematic reviews (ROBIS), Preferred Reporting Items for Systematic reviews and Meta-Analyses (PRISMA), and the grading of recommendations assessment, development, and evaluation (GRADE), respectively. Besides, the effectiveness and safety of Tai Chi for COPD were narratively summarized.

\section{Method}

\section{Protocol and Registration}

A priori protocol has been registered in the PROSPERO International Prospective Register of Systematic Reviews (registration number: CRD42019126600).

\section{Search Strategy}

We searched the following electronic bibliographic databases: PubMed, Embase, The Cochrane Library, China National Knowledge Infrastructure (CNKI), Wan Fang database and VIP database, using the keywords of Tai Chi, chronic obstructive pulmonary disease, and systematic review from inception to April 17, 2021. Studies published in English and Chinese were included. The search strategies were listed in Appendix A. In addition, we manually searched the references and relevant domestic journals, including the Chinese Journal of Rehabilitation Medicine, Chinese Journal of Rehabilitation Theory and Practice, and the Chinese Journal of Physical Medicine and Rehabilitation.

\section{Inclusion and Exclusion Criteria}

Inclusion criteria were established as follows: i) types of study: SRs containing more than one randomized controlled trial (RCT); ii) participants: Patients with COPD defined by spirometry; iii) interventions: Any type of Tai Chi with or without routine treatments; iv) comparison intervention: Routine treatments, such as drug therapy, routine activities, and respiratory training; v) outcome indicators: Pulmonary function including forced expiratory volume in 1 second (FEV1), FEV1\% predicted normal values (FEV1\%), the ratio of FEV1 to forced vital capacity [FEV1/FVC (\%)], 6-minute walking distance (6MWD) and the St. George's Respiratory Questionnaire (SGRQ). Exclusion criteria were: i) Guidelines, review comments, overviews of SRs and editorials. ii) Full text unavailable after contacting the author. Literature retrieval was done independently by two researchers (LPY/LYX).

\section{Data Management and Data Collection}

Endnote X7 (BId 7072) was used to perform data management. Two researchers (YZ/YLZ) independently screened titles and abstracted for potentially relevant studies after eliminating duplications. Full texts were downloaded and read for eligible included studies based on inclusion and exclusion criteria. Then a cross-check after completion was performed to avoid mis-entry. Any discrepancies were discussed by a third reviewer (JRJ).

Data extraction was independently completed by two researchers (YZ/YLZ). Discrepancies were discussed with a third reviewer (JL). A data extraction form was developed in advance. The main contents of data extraction were as follows: 
- General information (title, author, country, and language).

- Study characteristics (numbers of included RCTs, quality assessment tool, interventions and comparisons, data analysis methods).

- Outcomes: effect value, 95\% CI, p-value.

- Summary of conclusions.

\section{Assessment of Methodological Quality}

AMSTAR 2 is a common instrument to evaluate the methodology of SRs. ${ }^{18}$ Two researchers (LPY/DLZ) independently assessed the methodology of SRs using AMSTAR2. AMSTAR2 has 16 items, including seven critical items (item 2/4/7/9/11/13/15) which can critically affect the validity of a SR and its conclusion. Each item was evaluated as "yes," "partial yes," and "no" according to the standard of AMSTAR 2 guideline. ${ }^{18}$ The details of AMSTAR 2 items and the general rules for results of a $\mathrm{SR}$ are seen in Appendix B. Discrepancies were solved by team discussion.

\section{Assessment of Risk of Bias}

ROBIS is a tool designed specifically to assess the risk of bias in SRs, ${ }^{19}$ which is comprised of three phases formed by signaling questions. Two researchers (WW/TYL) independently assessed the risk of bias of each SR using ROBIS. Phase 1 assesses the assessing relevance, which is optional. Phase 2, which is comprised of four domains formed by 21 signaling questions, aims to identify concerns with the review process. Phase 3, with three signaling questions, concentrates to judge risk of bias of the SR. All signaling questions were answered as "yes", "probably yes", "probably no", "no", and "no information". If all of signaling questions of phase 3 were answered as "yes," SR was judged as "low risk". Any of signaling question of phase 3 was answered as "probably no" or "no", SR was assessed as "high risk". If the information provided was insufficient to judge, SR was rated as "unclear risk". Discrepancies were solved by team discussion.

\section{Assessment of Reporting Quality}

PRISMA is a reporting guideline designed to improve transparency of SRs, ${ }^{20-22}$ which consisted of a 27 -item checklist and a 4-phase flow diagram. Two researchers (YXL/LG) independently assessed reporting quality of included SRs using PRISMA. The highest score is 27. Each item was answered as "yes", "no", and "not applicable". We assessed the completion of the overall SRs' reporting by using a percentage, which was obtained through summing all items' score and dividing by the maximum score. The completion for each item was presented in ratio. Discrepancies were solved by team discussion.

\section{Assessment of Quality of Evidence}

GRADE is widely used for rating the quality of evidence of each outcome in SRs. ${ }^{23}$ Two researchers (ZDL/LPY) independently utilized the GRADE tool to assess quality of each outcome in included SRs, including FEV1, FEV1/ FVC (\%), FEV1\%, 6MWD, and SGRQ. According to GRADE, evidence based on RCTs begin as high quality, but the quality of evidence may be downgraded dependent on five key factors (Risk of Bias, Inconsistency, Indirectness, Imprecision and Publication bias) of GRADE. Quality of evidence of each outcome was rated as "High", "Moderate", "Low", and "Very Low".

\section{Data Synthesis and Presentation}

The results of AMSTAR 2, ROBIS, and PRISMA were summarized as a percentage of achievement per item. The results of AMSTAR 2, ROBIS, PRISMA, and the GRADE were summarized via tabulations. The characteristics of included SRs, the effectiveness and safety of Tai Chi for COPD were narratively summarized.

\section{Results}

\section{Results on Literature Search and Selection}

We retrieved 101 citations and excluded 37 duplicates before screening. The 43 citations were excluded by title and abstract screening. After reading full-text, 12 eligible articles were included. A flow diagram of literature search was shown in Figure 1.

\section{Characteristics of Included Reviews}

Of these 12 SRs, six ${ }^{24-29}$ studies were written in Chinese and six ${ }^{11,30-34}$ in English. They were published from 2013 to 2021. As for intervention, eight SRs took Tai Chi combined with other treatments (eg, drug treatment, routine rehabilitation therapy) as treatment, and four included Tai Chi alone. Several common treatments were used as comparison, including drug therapy, routine activities, respiratory training, aerobics, strength training, etc. The outcomes of the 12 studies included pulmonary function [FEV1, FEV1\%, FEV1/FVC (\%)], 6MWD, and SGRQ. In 




Figure I Flow diagram of literature search.

addition, seven ${ }^{11,24,27,29-31,33}$ of these SRs used The Cochrane Collaboration's Risk of Bias to assess the risk of bias of the original RCTs. The remaining four ${ }^{25,26,28,32}$ SRs used Jadad scores and one $\mathrm{SR}^{34}$ used the Physiotherapy Evidence Database (PEDro) scale. Details are shown in Table 1.

\section{Results of Methodological Quality}

Table 2 shows the results of methodological quality of the included SRs using AMSTAR 2. Among the 12 included SRs, 10 SRs were considered critically low quality and two SRs ${ }^{11,30}$ were low quality. AMSTAR 2 items(I) (ie, lower rates of "yes") with the lowest compliance rates were I2 ("a prior protocol provided", 8.33\%), I3 (Did the review authors explain their selection of the study designs for inclusion in the review?, 0\%), I5 (Did the review authors perform study selection in duplicate?, $42 \%$ ), I7
(Did the review authors provide a list of excluded studies and justify the exclusions?, 17\%), I12 ("the potential impact of RoB in individual studies on the results", $42 \%$ ), I13 ("Did the review authors account for RoB in primary studies when interpreting/discussing the results of the review?", 42\%), and I15 ("publication bias assessed", 42\%).

\section{Results of Risk of Bias}

According to Table 3, all SRs were in low risk in Phase 1. In phase 2, low risk of domain 1 was $100 \%$, domain 2 was $33.33 \%$, domain 3 was $66.67 \%$, and domain 4 was $66.67 \%$. Low risk of phase 3 was $33.33 \%$. In addition, signaling questions $(\mathrm{Q})$ of ROBIS with the highest concerns (ie, higher rates of "no") were Q6 (Did the search include an appropriate range of databases/electronic sources for published and unpublished reports?, 


\begin{tabular}{|c|c|c|c|c|c|}
\hline 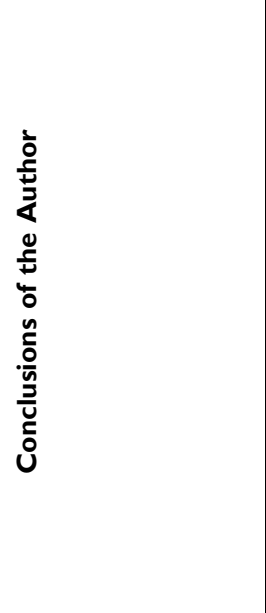 & 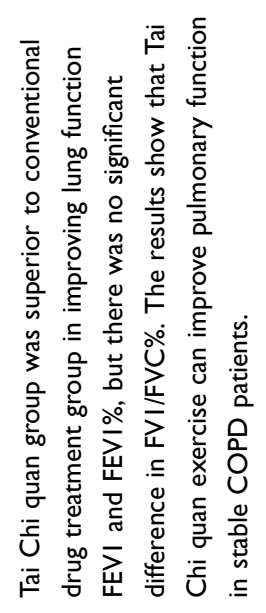 & 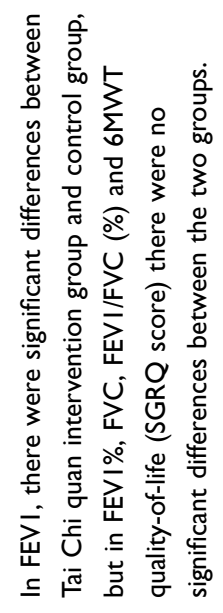 & 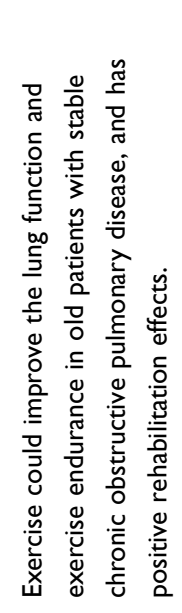 & 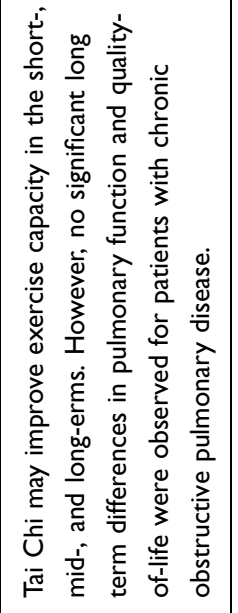 & 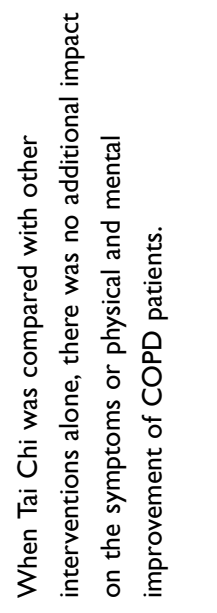 \\
\hline 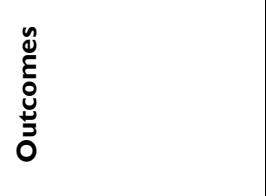 & 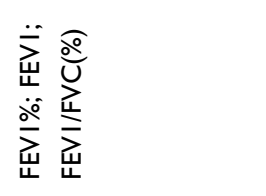 &  & 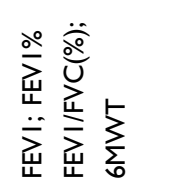 & 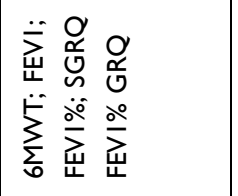 & 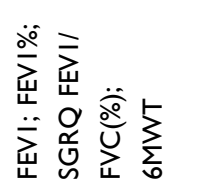 \\
\hline  & 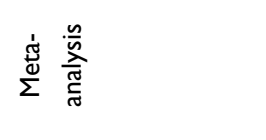 & 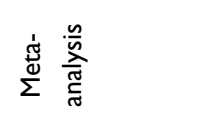 & 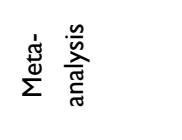 & 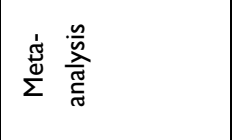 & 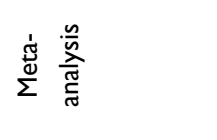 \\
\hline 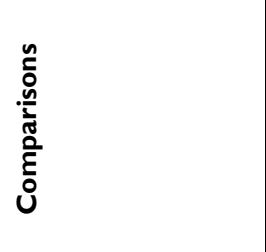 & 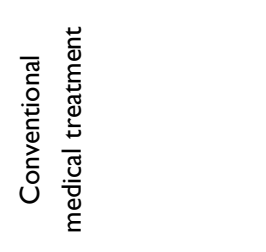 & 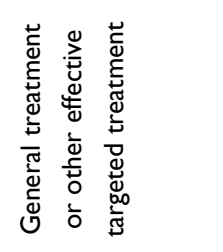 &  & 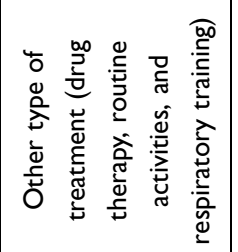 & 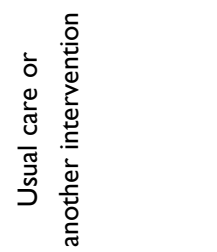 \\
\hline 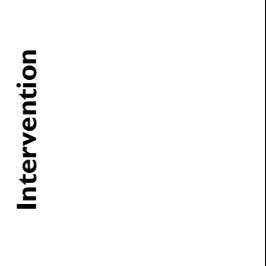 & 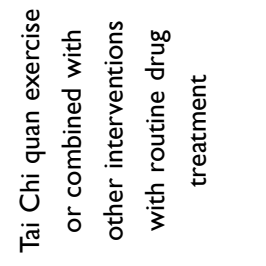 &  & 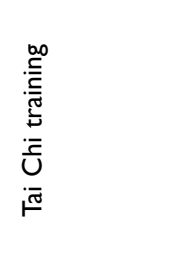 &  & \\
\hline  & 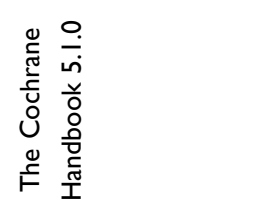 &  &  &  & 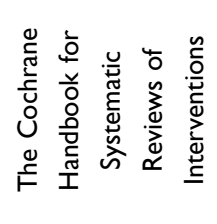 \\
\hline 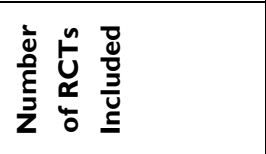 & $\begin{array}{l}\stackrel{a}{U} \\
a \\
\sigma\end{array}$ & $\begin{array}{l}\tilde{U} \\
\propto \mathscr{u} \\
n\end{array}$ &  & $\mid \begin{array}{l}\underline{\underline{U}} \\
\alpha \\
\underline{\underline{n}}\end{array}$ & $\begin{array}{l}\underset{U}{U} \\
\stackrel{\sim}{\simeq} \\
\end{array}$ \\
\hline 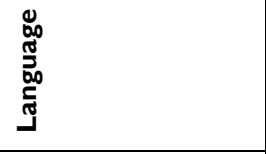 & 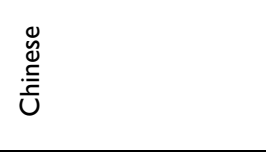 & 岕 & $\begin{array}{l}\mathscr{\Xi} \\
\stackrel{\mathscr{E}}{\bar{E}} \\
\end{array}$ & 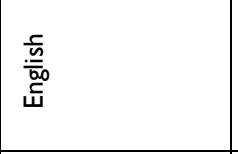 & 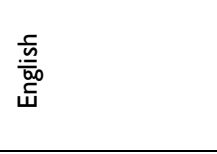 \\
\hline 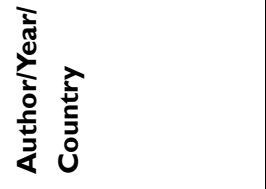 & 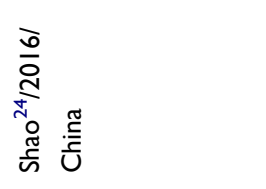 &  & 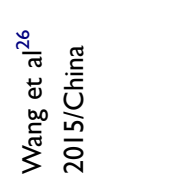 & 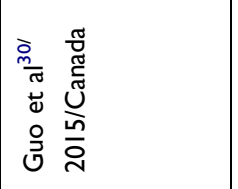 & 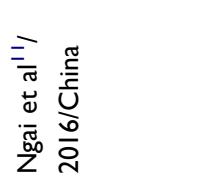 \\
\hline
\end{tabular}




\begin{tabular}{|c|c|c|c|c|c|c|c|}
\hline  & 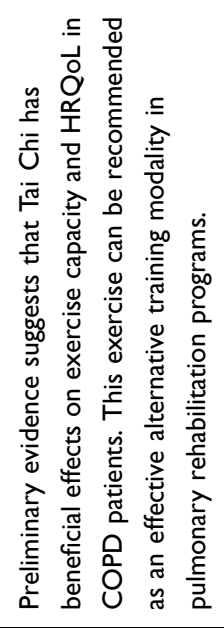 &  & 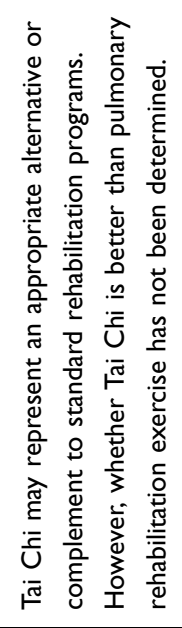 & 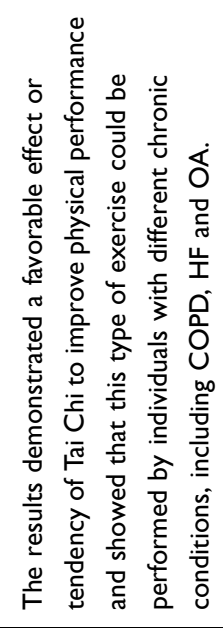 &  & 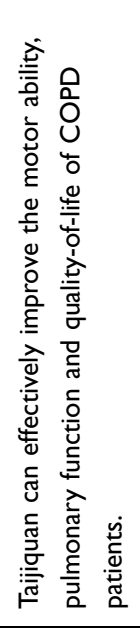 & 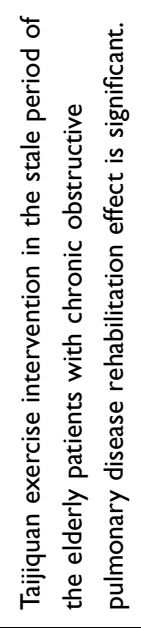 \\
\hline $\begin{array}{l}\stackrel{y}{E} \\
\stackrel{0}{0} \\
\stackrel{u}{0} \\
0\end{array}$ & 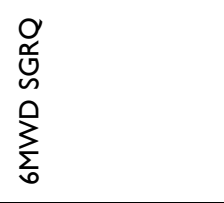 &  & 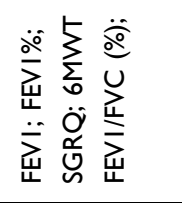 & $\sum_{i}^{5}$ & 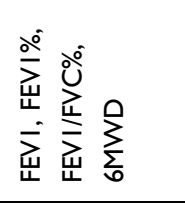 & 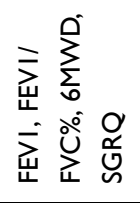 & $\sum_{i}^{0}$ \\
\hline 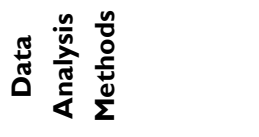 & 总 & 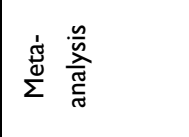 & 离 & 离 & 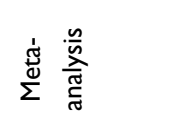 & 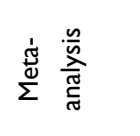 &  \\
\hline  & 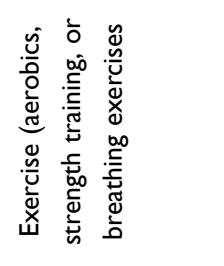 & 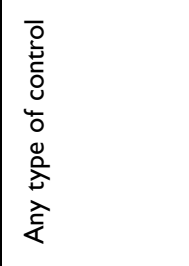 & 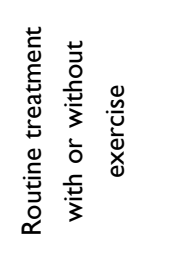 & 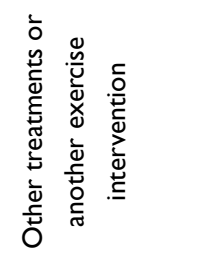 &  & 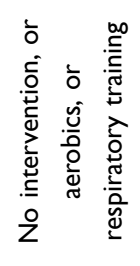 & 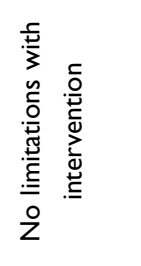 \\
\hline 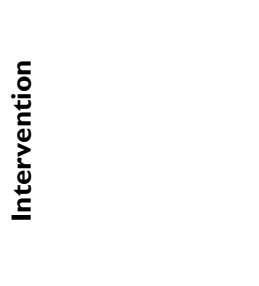 & $\begin{array}{l}\dot{\bar{U}} \\
\dot{\bar{U}} \bar{\sigma}\end{array}$ & 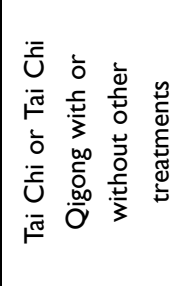 & 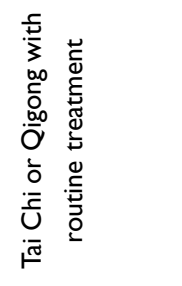 & 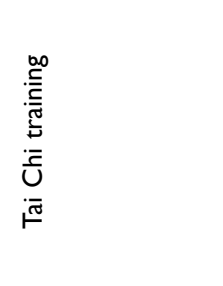 & 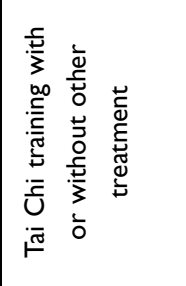 & 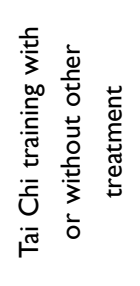 & 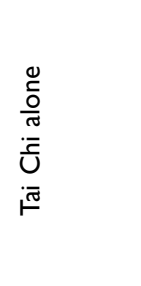 \\
\hline  & 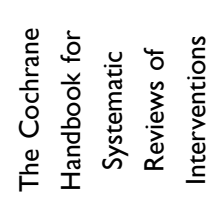 & 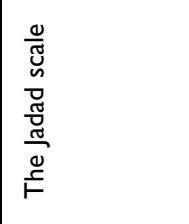 & 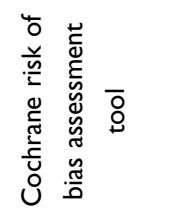 &  & 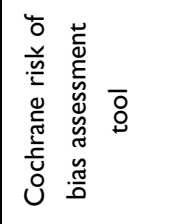 &  & 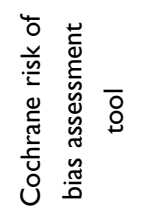 \\
\hline  & $\begin{array}{l}\underset{\mathscr{U}}{\simeq} \\
=\end{array}$ & $\mid \begin{array}{l}\tilde{y} \\
\frac{n}{\alpha} \\
\infty\end{array}$ & $\begin{array}{l}\underline{\underline{v}} \\
\stackrel{x}{0} \\
\underline{0}\end{array}$ & $\begin{array}{l}\tilde{U} \\
\alpha \\
\sigma\end{array}$ & 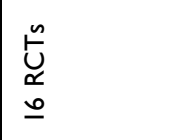 & $\begin{array}{l}\underset{\mathscr{U}}{\sigma} \\
\sigma\end{array}$ & $\begin{array}{l}\underline{\underline{u}} \\
\propto \sim \\
\underline{n}\end{array}$ \\
\hline  & $\begin{array}{l}\frac{\bar{c}}{\frac{\underline{\underline{m}}}{w}} \\
\underline{w}\end{array}$ & 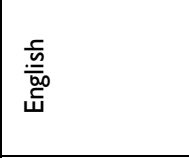 & $\begin{array}{l}\frac{5}{\frac{5}{b 0}} \\
\frac{w}{w}\end{array}$ & $\begin{array}{l}\frac{c}{\frac{\underline{m}}{w 0}} \\
\underline{w}\end{array}$ & 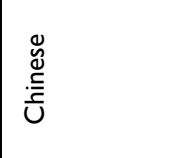 & 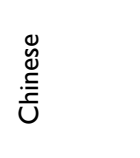 & 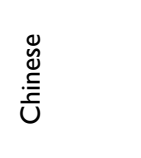 \\
\hline 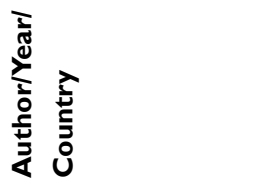 & 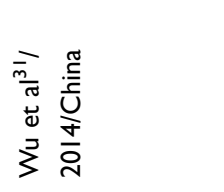 & 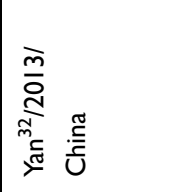 & 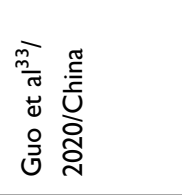 &  &  & 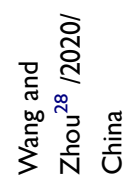 & 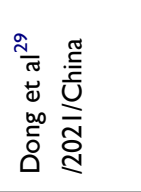 \\
\hline
\end{tabular}




\begin{tabular}{|c|c|c|c|c|c|c|c|c|c|c|c|c|c|c|c|c|c|}
\hline  & $\succ$ & Z & Z & Z & Z & $\succ$ & $\mathbf{Z}$ & $\succ$ & $\succ$ & $\succ$ & $\succ$ & Z & Z & $\succ$ & Z & Z & 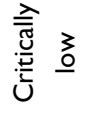 \\
\hline 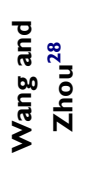 & $\succ$ & Z & Z & $\succ$ & $\succ$ & Z & $\mathbf{Z}$ & $\succ$ & $\succ$ & $\succ$ & $\succ$ & Z & Z & $\mathbf{Z}$ & Z & Z & 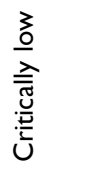 \\
\hline 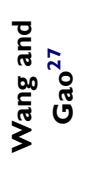 & $\succ$ & Z & Z & Z & $\succ$ & $\succ$ & $\mathbf{Z}$ & $\succ$ & $\succ$ & $\succ$ & $\succ$ & $\mathbf{Z}$ & Z & $\succ$ & $\succ$ & Z & 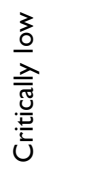 \\
\hline 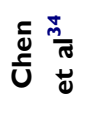 & $\succ$ & Z & Z & Z & $\succ$ & $\succ$ & Z & $\succ$ & $\succ$ & $\succ$ & $\succ$ & Z & Z & $\succ$ & $\succ$ & $\succ$ &  \\
\hline
\end{tabular}

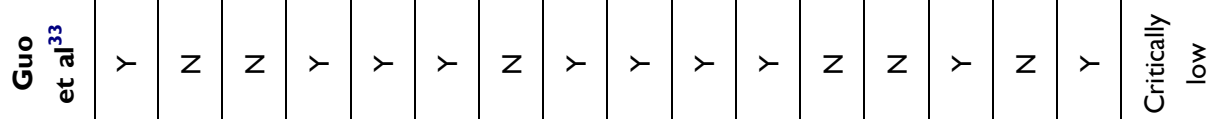

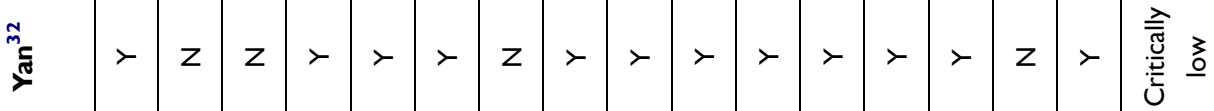

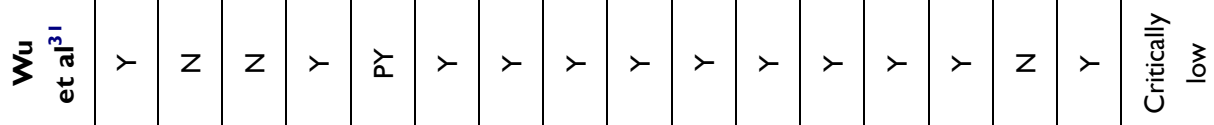

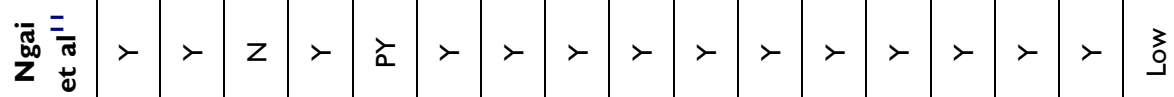

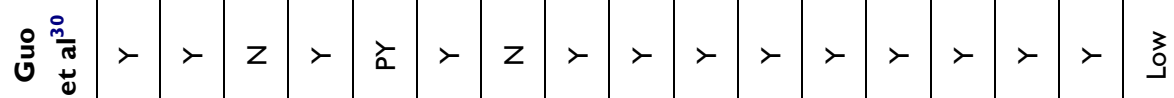

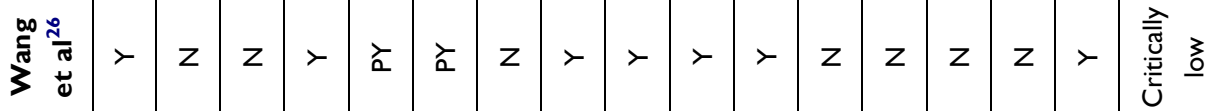

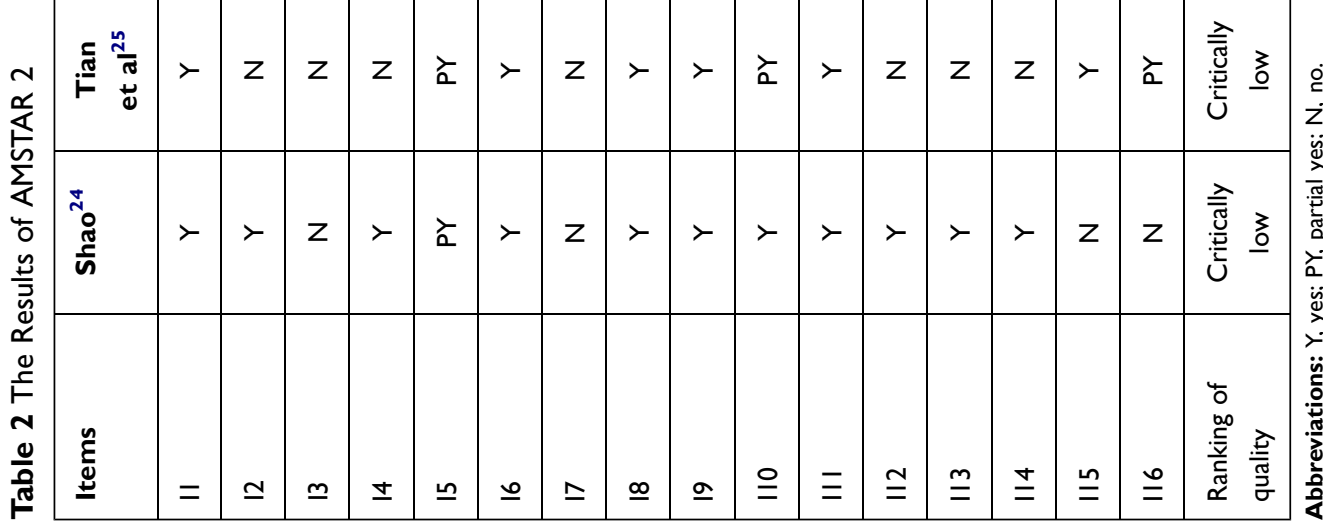




\begin{tabular}{|c|c|c|c|c|c|c|c|c|c|c|c|c|c|}
\hline 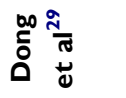 & $\succ$ & え & $\succ$ & $\succ$ & $\succ$ & $\succ$ & $\underbrace{3}_{0}$ & $\succ$ & $z$ & $\bar{z}$ & $\bar{z}$ & $z$ & 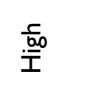 \\
\hline 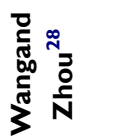 & $\succ$ & $\check{a}$ & $\succ$ & $\succ$ & $\succ$ & $\succ$ & 3 & $\succ$ & $z$ & $\succ$ & $\succ$ & $\succ$ & $\begin{array}{l}\frac{\vec{c}}{\mathrm{~b} 0} \\
\frac{1}{I}\end{array}$ \\
\hline 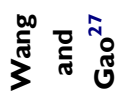 & $\succ$ & こ & $\succ$ & $\succ$ & $\succ$ & $\succ$ & 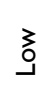 & z & えั & $z$ & $\check{\alpha}$ & $\succ$ & $\frac{\frac{\bar{b}}{60}}{\bar{I}}$ \\
\hline 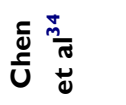 & $\succ$ & え & $\succ$ & $\succ$ & $\succ$ & $\succ$ & 芆 & $\succ$ & $\succ$ & $\succ$ & $z$ & $\succ$ & ב. \\
\hline$\stackrel{\circ}{\stackrel{m}{\frac{m}{\sigma}}}$ & $\succ$ & ¿ & $\succ$ & $\succ$ & $\succ$ & $\succ$ & $\underbrace{3}_{0}$ & $\succ$ & $z$ & $\succ$ & $\succ$ & $\succ$ & $3_{3}^{3}$ \\
\hline 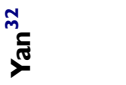 & $\succ$ & $\grave{a}$ & $\succ$ & $\succ$ & $\succ$ & $\succ$ & 3 & $z$ & $z$ & $z$ & $\succ$ & $\succ$ & $\frac{\frac{c}{b 0}}{\bar{I}}$ \\
\hline$\sum^{\bar{\xi}} \stackrel{\bar{m}}{\bar{\sigma}}$ & $\succ$ & え & $\succ$ & $\succ$ & $\succ$ & $\succ$ & 艿 & $\succ$ & $\succ$ & $\succ$ & $\succ$ & $\bar{z}$ & 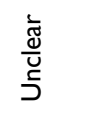 \\
\hline $\begin{array}{l}\text { 要 } \\
\bar{z} \\
\bar{z} \\
\overline{0}\end{array}$ & $\succ$ & خ̀ & $\succ$ & $\succ$ & $\succ$ & $\succ$ & ?3 & $\succ$ & $\succ$ & $\succ$ & $\succ$ & $\succ$ & 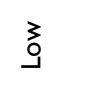 \\
\hline $\begin{array}{l}\frac{0}{\frac{1}{\pi}} \\
\stackrel{5}{0} \\
0 \\
0 \\
0 \\
0\end{array}$ & $\succ$ & $\succ$ & $\succ$ & $\succ$ & $\succ$ & $\succ$ & ${ }_{0}^{3}$ & $\succ$ & $z$ & $\succ$ & $\succ$ & $\bar{z}$ & 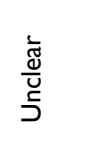 \\
\hline 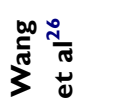 & $\succ$ & え & $\succ$ & $\succ$ & $\succ$ & $\succ$ & ?3 & $z$ & $z$ & $z$ & $\succ$ & $\succ$ & 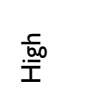 \\
\hline  & $\succ$ & $\grave{a}$ & $\succ$ & $\succ$ & $\succ$ & $\succ$ & $3_{0}^{3}$ & $z$ & $\succ$ & $z$ & $\succ$ & 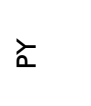 & $\frac{\sqrt{\frac{1}{b 0}}}{\frac{1}{I}}$ \\
\hline 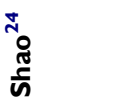 & $\succ$ & $\succ$ & $\succ$ & $\succ$ & $\succ$ & $\succ$ & 30 & $\succ$ & $\succ$ & $\succ$ & $\succ$ & $\succ$ & בُ \\
\hline 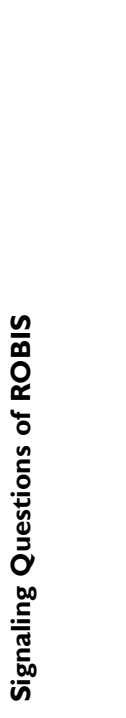 & 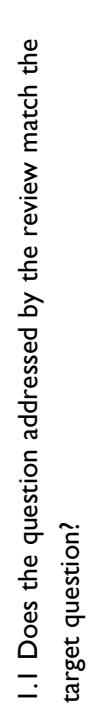 & 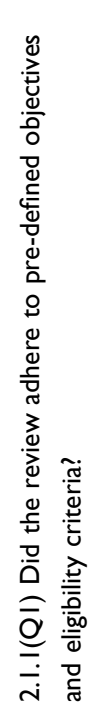 & 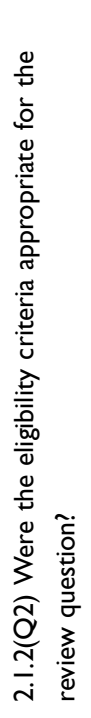 & 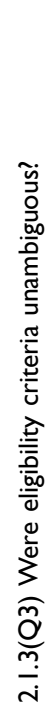 & 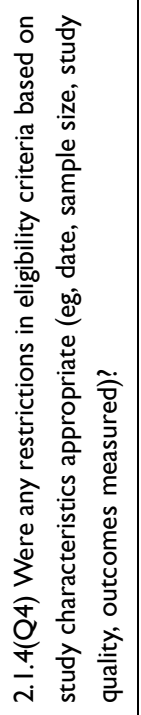 & 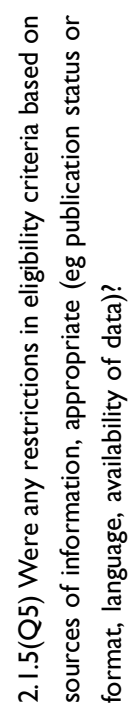 & 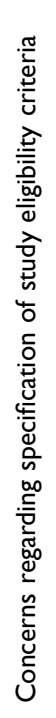 & 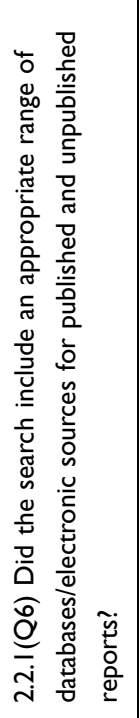 & 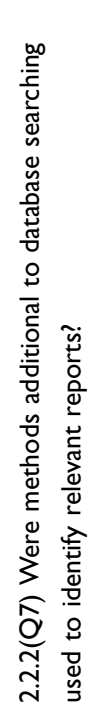 & 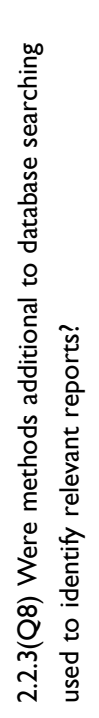 & 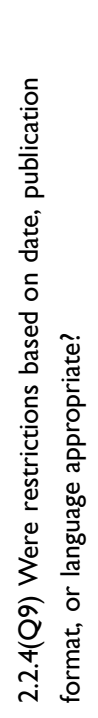 &  & 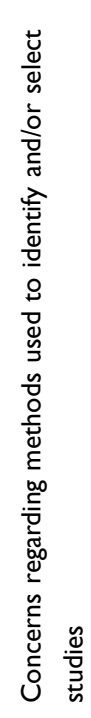 \\
\hline
\end{tabular}


Dovepress

Yang et al

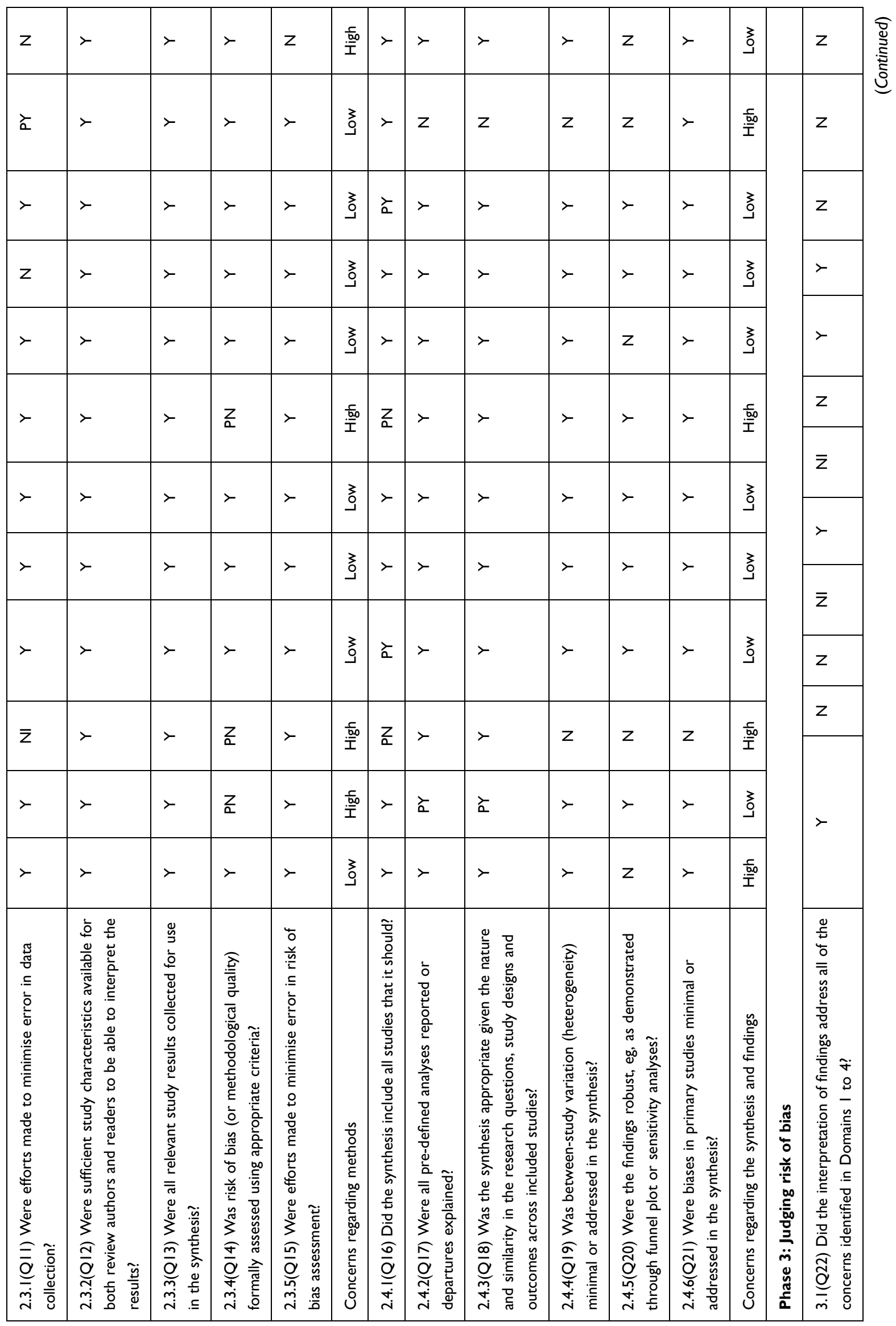

International Journal of General Medicine 2021:|4

https://doi.org// 0.2147/IJGM.S308955 $\mathbf{3 0 2 5}$ 


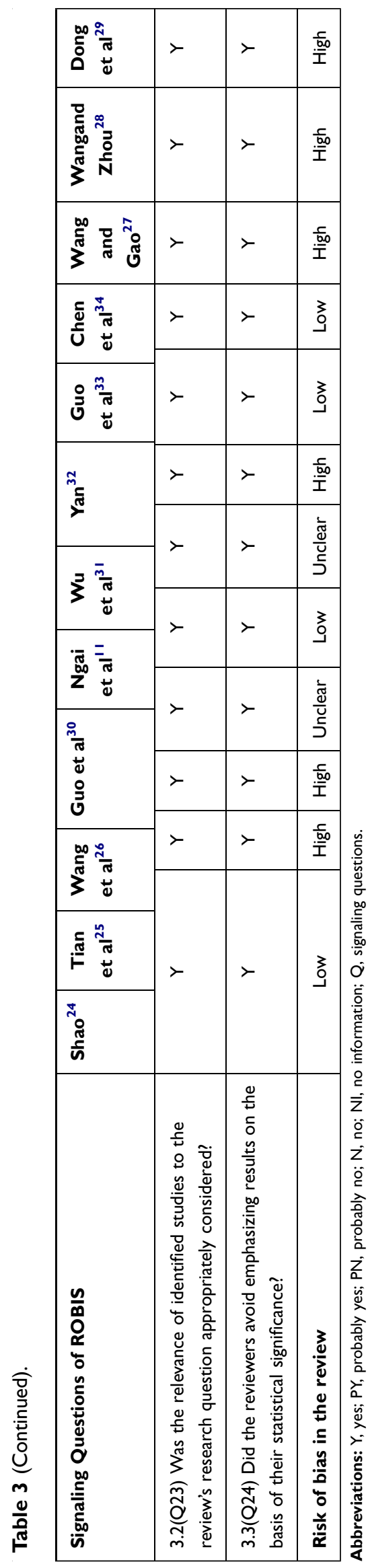

no $=33.33 \%$ ), Q7 (Were methods additional to database searching used to identify relevant reports?, no $=50 \%$ ), Q20 (Were the findings robust, eg, as demonstrated through funnel plot or sensitivity analyses. no $=41.67 \%$ ), and Q22 (Did the interpretation of findings address all of the concerns identified in Domains 1 to 4 ?, no $=50 \%$ ). According to the final phase of ROBIS, four out 12 of SRs was rated as "low risk", 6SRs were "High risk", and two SRs were "Unclear risk".

\section{Results of Reporting Quality}

We used PRISMA to assess the reporting quality of included SRs. We found that the section of title, abstract, introduction, study characteristics, limitation, conclusion, and funding were all well reported. We observed that nine out of 12 SRs were found adequately reported over $80 \%$. However, several items (I) had a lower score (ie, lower rates of "yes"), that were I5 (Protocol and registration, yes $=8.33 \%$ ), I9 (Study selection, yes $=41.67 \%$ ), I11 (Data items, yes $=25 \%$ ), I16 (Additional analyses, yes $=60 \%$ ), and I22 (Risk of bias across studies, yes $=25 \%$ ). These items may account for the main reporting limitations and should be paid attention. The PRISMA checklists of each SR were presented in Table 4.

\section{Results with GRADE Tool}

In our study, evidence of FEV1 in three SRs (3/12, $25 \%)^{11,24,30}$ was rated as "Moderate", noevidence of FEV1\% was rated as "Moderate", evidence of FEV1/ FVC (\%) in one SR $(1 / 12,9 \%)^{11}$ was rated as "Moderate", evidence of 6MWD in three SRs ${ }^{11,30,31}$ was rated as "Moderate", evidence of SGRQ in two $\mathrm{SRs}^{28,33}$ was rated as "Moderate", evidence of FEV1/ FVC (\%) in one SR $(1 / 12,9 \%)^{11}$ was rated as "Moderate", and the remaining evidence was rated as "Low" or "Very Low". We presentthem in Table 5. Deserved to be mentioned, Tai Chi is a physical exercise which consists of different forms and postures, it is not practical for participants to be blinded about group allocation. Lack of blinding may lead to bias. Thus, all studies were graded as "serious $(-1)$ " in category of risk of bias. This may be the reason why there was no highquality evidence in our study. Besides, the quality of the same outcome among different the included SRs are inconsistent, a comprehensive and rigorous SR is needed to verify the effectiveness of Tai Chi for COPD. 


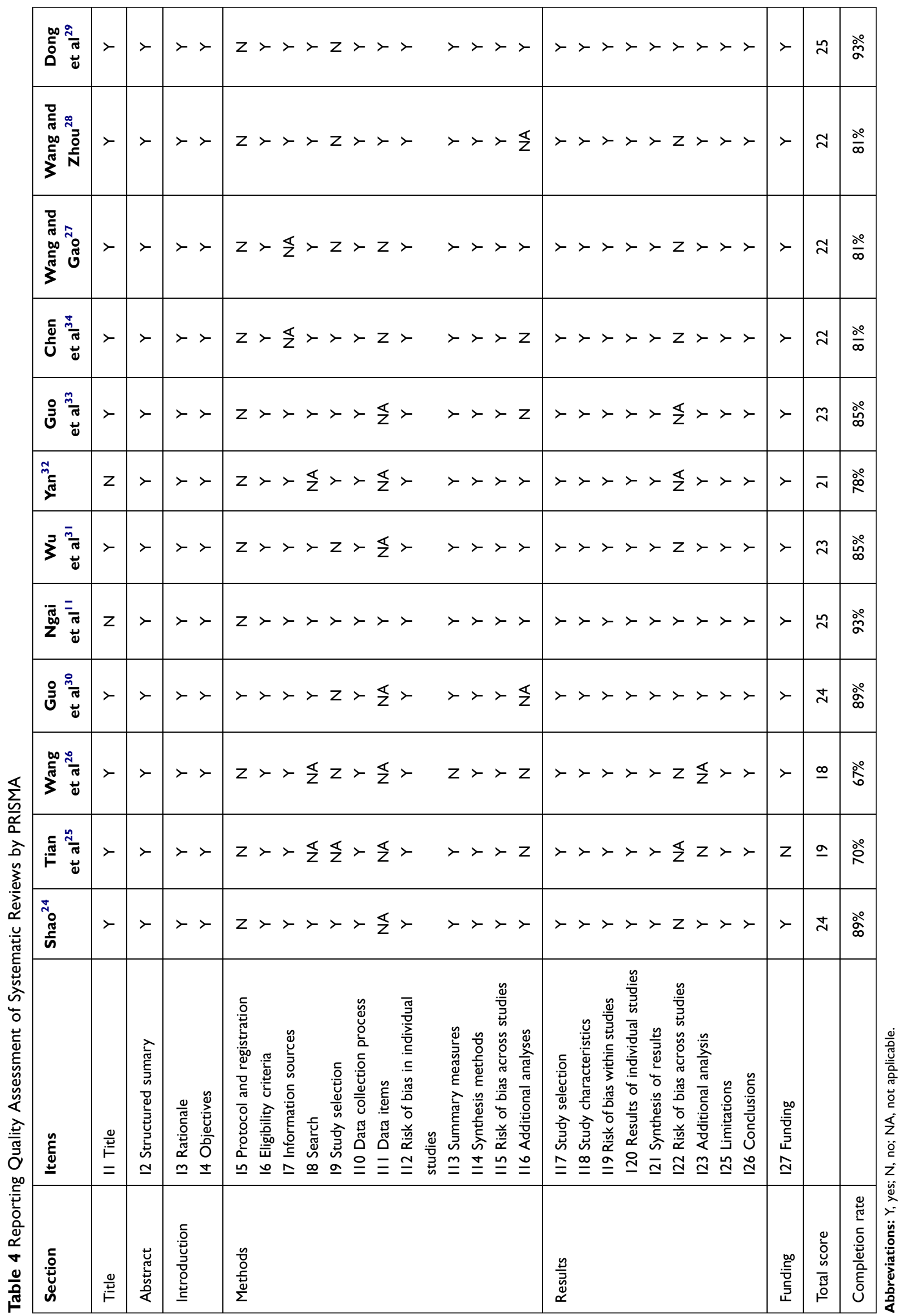




\begin{tabular}{|c|c|c|c|c|c|}
\hline 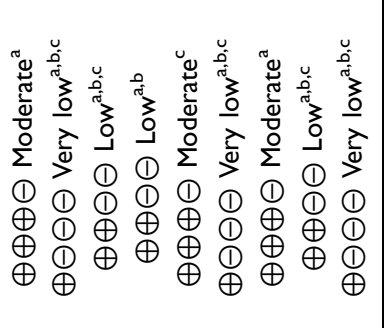 &  &  & 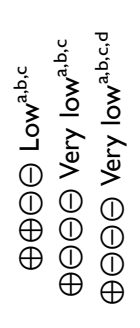 & 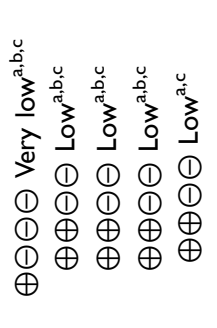 & 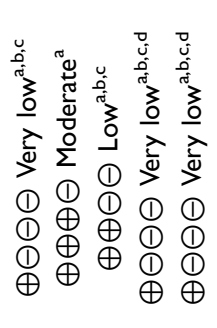 \\
\hline 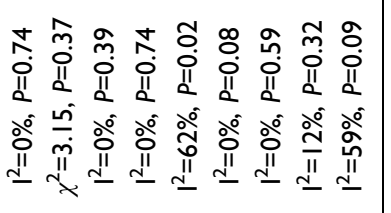 & 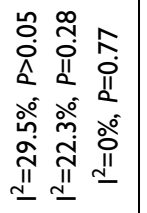 & 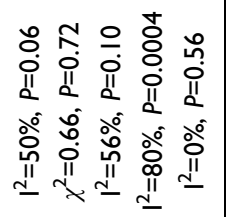 &  & 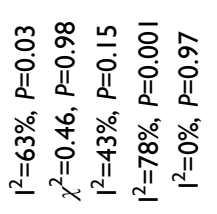 & 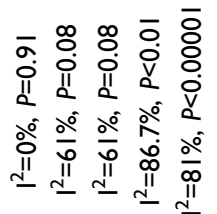 \\
\hline  & 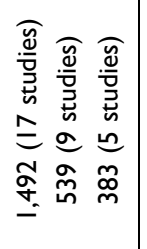 &  &  & 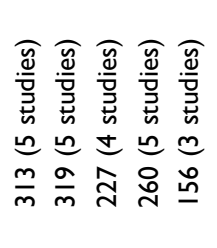 & 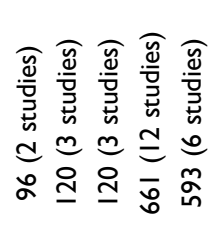 \\
\hline 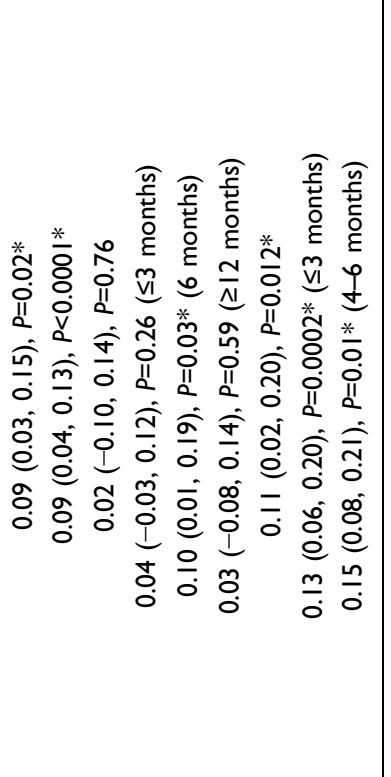 & 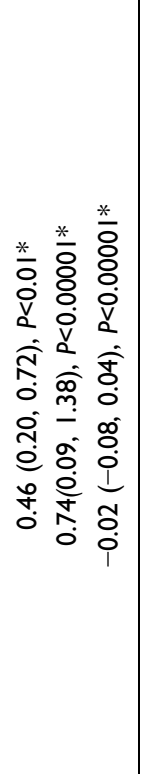 & 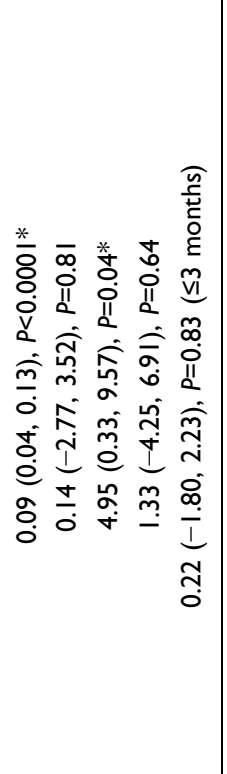 & 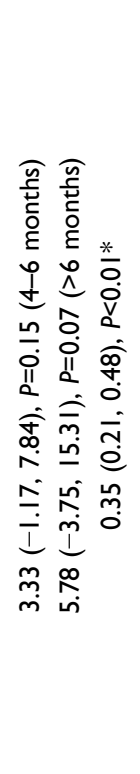 & 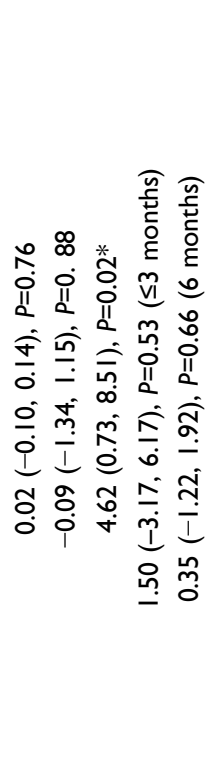 & 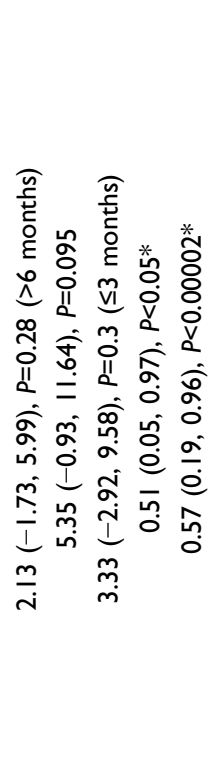 \\
\hline 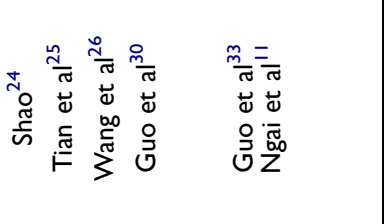 & 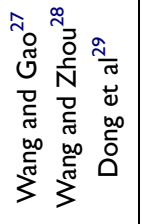 & 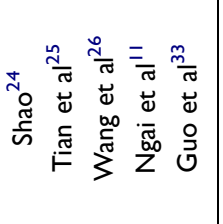 & 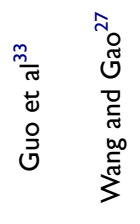 & 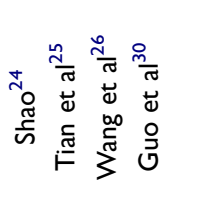 & 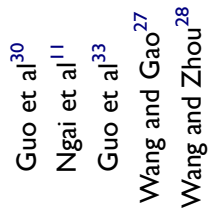 \\
\hline 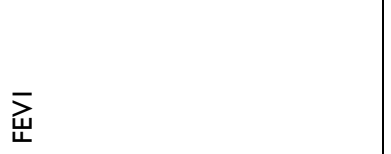 & 蛋 & 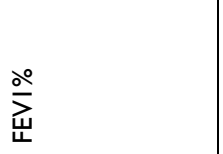 & $\sum_{\frac{\mathrm{j}}{u}}^{\circ 0}$ & 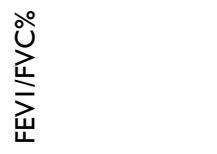 & 总 \\
\hline
\end{tabular}




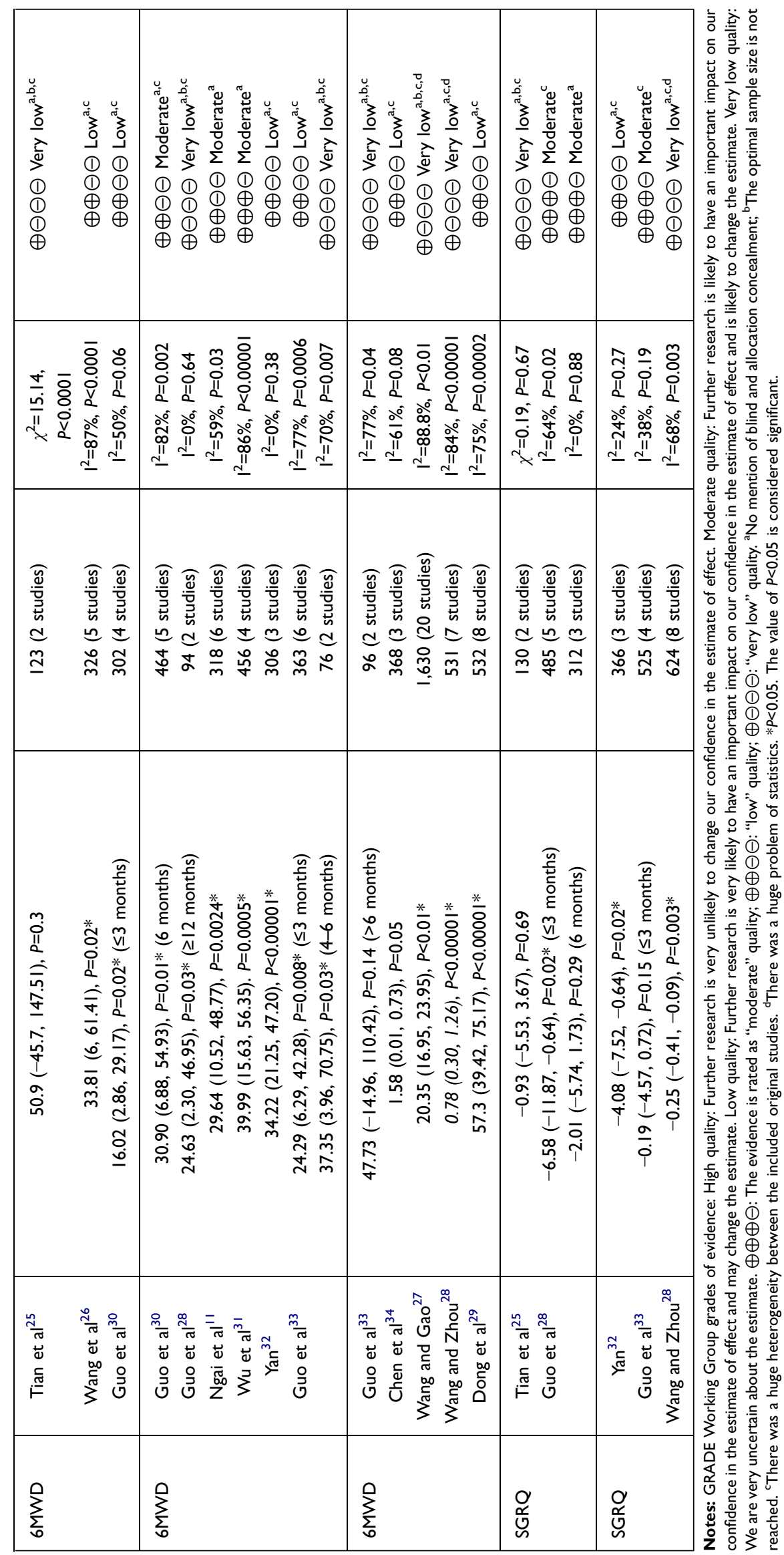




\section{The Effectiveness and Safety of Tai Chi}

We summarized the information from included SRs and found that their results were inconsistent. The details are shown in Table 5. FEV1, as we all know, has always been used as a primary metric of severity for obstructive lung disease. ${ }^{35,36}$ Among the SRs, seven out of nine SRs (78\%) reported that FEV1 was significantly enhanced in the Tai Chi group, and one SR (11\%) reported not. One SR (11\%) reported that FEV1 was significantly improved after 6months of Tai Chi training, but with no significance less than 3 months or over 12 months.

Both FEV1\% and FEV1/FVC\% were the main indicators for the diagnosis of COPD. ${ }^{35,37}$ According to the results of FEV1\%, three out of six SRs (50\%) reported that FEV1\% was significantly enhanced in the Tai Chi group, and three SRs (50\%) reported not. As for FEV1/ FVC\%, three out of eight SRs (38\%) reported that FEV1/ FVC\% was significantly enhanced in the Tai Chi group, five SRs $(62 \%)$ reported that Tai Chi had no priority in FEV1/FVC\%.

6MWD is a simple and reproducible test aiming to a global and integrated response of both physical (pulmonary and non-pulmonary factors) and psychological factors. ${ }^{38-40}$ It can be used to assess the functional exercise capacity. ${ }^{41}$ Of the 12 SRs, nine (75\%) reported that 6MWD was significantly enhanced in the Tai Chi group. Only one SR (8\%) reported that Tai Chi had no priority, and one SR (7\%) reported that there was a significant increase at 3 months and 6 months, but no significance over 6 months.

SGRQ is a widely used instrument that is able to assess the health status and health-related quality-of-ife in COPD. ${ }^{42,43}$ Among the five included SRs, three SRs $(60 \%)$ reported that SGRQ was significantly enhanced in the Tai Chi group. Two SRs (40\%) reported that Tai Chi had no priority in SGRQ.

Only two SRs made subgroup analysis based on the duration and the remaining SRs had an ambiguous description of duration for Tai Chi. We found that the duration of Tai Chi affected the results of SR. For example, Guo et $\mathrm{al}^{30}$ found that FEV1 was significant increased after 6-months of Tai Chi training, but had no significance less than 3 months or over 12 months. Guo et al ${ }^{33}$ discovered that FEV1 could be improved after 3-months of Tai Chi training.

Among the 12 included studies, the intervention in the experimental group in nine SRs was Tai chi combined other rehabilitation therapy, only three SRs adopted Tai Chi alone. Wu et $\mathrm{al}^{31}$ and Chen et $\mathrm{al}^{34}$ only reported the results of $6 \mathrm{MWD}$. However, they got opposite results. $\mathrm{Wu}$ et $\mathrm{al}^{31}$ reported that $6 \mathrm{MWD}$ was significantly enhanced in the Tai Chi group, but Chen et $\mathrm{al}^{34}$ observed no significant difference. The result of 6MWD in a study of Dong et $\mathrm{al}^{29}$ was consistent with $\mathrm{Wu}$ et al. ${ }^{31}$ Dong et $\mathrm{al}^{29}$ also reported that FEV1 was not significantly enhanced in the Tai Chi group. In addition, among the 12 included SRs, no adverse effects of Tai chi on COPD were reported.

\section{Discussion}

\section{The Main Findings}

We narratively summarized the results of included SRs, and appraised the methodological quality, risk of bias, reporting quality, and quality of evidence of these SRs. Among the 12 SRs, 10 were considered as "critically low" in methodological quality and two ${ }^{11,30}$ were low quality according to AMSTAR 2. By the ROBIS, six SRs were rated as "High" risk of bias, four were "Low" risk, and two were "Unclear risk". With the PRISMA checklist, we found most of the SRs (75\%) were of relatively good reporting quality. However, the methodological quality, risk of bias, reporting quality, and quality of evidence of these SRs on Tai Chi for COPD were still suboptimal. Due to these limitations, the reliability of evidence for Tai Chi on COPD was weakened, and we should be cautious when recommending Tai $\mathrm{Chi}$ as an alternative treatment or alone treatment for improving pulmonary function and quality-of-life on COPD.

In addition, in this present study, we mainly focus on the methodology, risk of bias, and reporting quality of SRs about Tai Chi on COPD. We found that some items of AMSTAR 2, ROBIS, and PRISMA, may be repetitive. So, according to the results, we highlighted several common weaknesses of the included SRs as follows: 1) absence of a protocol registration or publication statement prior to the conduct of SR; 2) lack of a comprehensive literature search strategy and some additional methods for retrieving; 3) lack of appropriate instruments to assess the risk of bias (or methodological quality); 4) lack of prospectively describing the additional analyses in method; an 5) lack of appropriate methods to solve the heterogeneity and examine the robustness of the findings.

\section{Implications for Clinicians and Producers of Further Study For Clinicians}

Firstly, according to our results, Tai Chi was a safety treatment for COPD patients as no adverse events were reported in the included SRs. Secondly, we found that Tai 
Chi is prone to improve the functional capacity and quality-of-life in COPD patients, rather than pulmonary function. $75 \%$ of included SRs reported the significant increase for $6 \mathrm{MWD}$ in Tai Chi group, $60 \%$ for SGRQ, $78 \%$ for FEV1, $50 \%$ for FEV1\%, and only $38 \%$ for FEV1/FVC $\%$. Thirdly, due to the limitation of these SRs and the inconsistent results, we should be cautious when recommending Tai Chi as an alternative treatment or alone treatment on COPD.

\section{For Producers of Further SR}

Most of these shortcomings can be avoided if the producers pay more attention to methodology, risk of bias, and standard reporting quality of SRs. Therefore, we have several suggestions for SR producers.

We suggest that the producers should register SR on registration platform like the international databases PROSPERO (https://www.crd.york.ac.uk/prospero/). Numerous studies have indicated that prospective registration may benefit the design, conduct, and reporting of SRs. ${ }^{44-46}$ For producers of SRs, it is important to perform additional methods to ensure that more eligible studies can be retrieved, such as citation searches, contacting experts, reference checking, and handsearching. A list of excluded studies is necessary, otherwise there may be a risk that they remain invisible and the impact of their exclusion from the review is unknown. Risk of bias (or methodological quality) should be formally assessed using appropriate criteria. Of the 11 SRs, four, using the Jadad Scale, were all rated as "probably no" for absence of allocation concealment by AMSTAR2. The Jadad score does not include allocation concealment which has been shown to be a major potential risk of bias in RCTs. ${ }^{47}$ In addition, we suggest authors prospectively describe the additional analyses in statistical method, such as sensitivity analyses, subgroup analyses, and meta-regression.

For results of SR, the authors should focus on heterogeneity and robustness of findings. Heterogeneity may arise as a result of clinical or methodological differences between studies. ${ }^{48}$ Between-study variation might be inspected either visually or through statistical tests. Visual inspection mainly refers to the examination of the forest plots and the degree of overlap of the associated confidence intervals. ${ }^{49}$ Statistical heterogeneity may be confirmed through statistical tests, such as the $I^{2}$ statistic. $^{50}$ The Cochrane Collaboration has proposed the use of four categories when interpreting heterogeneity based on $I^{2}$ as follows: $0-40 \%$ unimportant heterogeneity, $30-60 \%$ moderate, $50-90 \%$ substantial, and $75-100 \%$ considerable heterogeneity. ${ }^{51}$ As for inevitable heterogeneity, we suggest the authors of SRs to perform meta-regression or subgroup analyses. In addition, robustness of the findings, which is often examined by funnel plots. Heterogeneity, reporting bias, and chance may all lead to asymmetry funnel plots. ${ }^{52}$ Therefore, we suggest the authors of SRs to perform a sensitivity analysis or use the funnel plots to examine relationships between effect size and study size.

\section{Strengths and Limitations}

There are some strengths in our study. First, it is the first overview which comprehensively appraised the SRs of Tai Chi for COPD with AMSTAR 2, ROBIS, PRISMA, and GRADE. It can help the physicians and patients to have a better understanding of the effectiveness on Tai Chi for COPD in clinical practice. Second, in order to reduce the risk of bias in the review, we prospectively registered on the PROSPERO platform to ensure the transparency of the study. Despite the advantages, this study also had some limitations. First, there might be some missing information since we only searched studies in English and Chinese language. Second, since there are overlapping studies between the included SRs, we only made narratively described the effectiveness and safety of Tai Chi on COPD, but did not synthesize the data from original RCT included in eligible SRs.

\section{Conclusion}

There are more and more SRs concerning the Taiji treatment of COPD. However, through assessing the current SRs on Tai Chi for COPD, we found that the methodological quality, risk of bias, reporting quality, and quality of evidence of these SRs were suboptimal. Further well-conducted SRs with less risk of bias, more rigorous methodology, normative reporting, and high-quality of evidence are needed to provide robust evidence on Tai Chi for COPD.

\section{Abbreviations}

COPD, chronic obstructive pulmonary disease; CNKI, China National Knowledge Infrastructure; VIP database, Wei Pu database; RCT, randomized controlled trials; SR, systematic review; FEV1, forced expiratory volume in one second; FVC, forced vital capacity; 6MWD, 6-minute walking distance; SGRQ, the St. George's Respiratory Questionnaire; ROBIS, risk of bias in systematic reviews; PRISMA, the Preferred Reporting Items for Systematic 
Reviews and Meta-Analyses; GRADE, the grading of recommendations assessment, development and evaluation; PICO, participants, interventions, controls, and outcomes; PEDro, the physiotherapy evidence database; MCID, the minimum clinically important difference; EQ5D, EuroQol-5 Dimension; SF-36, the 36-Item Short Form Health Survey.

\section{Ethics Approval and Consent to Participate}

Ethical assessment and informed consent were not required since our research did not involve individual data.

\section{Acknowledgments}

We would like to appreciate Mingxing He and Yi Jie Huang for their help on methodology in our manuscript. These authors contributed equally to this study and share first authorship: Luping Yang, Dongling Zhong, and Yue Zhang.

\section{Funding}

The publication fee of this study is financially supported by the National Natural Science Foundation of China (No. 81873356), National Key Research and Development Project (2019YFC1710302) and The Project of Scientific and Technical department in Sichuan (No. 81704137).

\section{Disclosure}

The authors declare no conflicts of interest for this work.

\section{References}

1. Global Strategy for the Diagnosis. Management and prevention of chronic obstructive pulmonary disease (2019 REPORT). the guide and the complete list of references examined by the committee are available on the GOLD. Available from: www.goldcopd.org. Accessed May 27, 2021.

2. Lopez AD, Shibuya K, Rao C, et al. Chronic obstructive pulmonary disease: current burden and future projections. Eur Respir J. 2006;27 (2):397-412. doi:10.1183/09031936.06.00025805

3. GBoDS Collaborators. Global, regional, and national age-sex specific all-cause and cause-specific mortality for 240 causes of death, 1990-2013: a systematic analysis for the Global Burden of Disease Study 2013. Lancet. 2015;385(9963):117-171. doi:10.1016/S01406736(14)61682-2

4. Celli BR. Management Guidelines for Chronic Obstructive Pulmonary Disease. In: Hanania N, Sharafkhaneh A (eds). COPD. Respiratory Medicine. Humana Press; 2011:81-98. https://doi.org/10.1007/978-159745-357-8_5

5. Gómez FP, Rodriguez-Roisin R. Global Initiative for Chronic Obstructive Lung Disease (GOLD) guidelines for chronic obstructive pulmonary disease. Curr Opin Pulm Med. 2002;8(2):81. doi:10.1097/ 00063198-200203000-00001

6. Hempel S, Taylor SL, Solloway MR, et al. Evidence Map of Tai Chi. Department of Veterans Affairs; 2014.
7. Lau J, Ioannidis JP, Schmid CH. Summing up evidence: one answer is not always enough. Lancet. 1998;351(9096):123-127. doi:10.1016/ S0140-6736(97)08468-7

8. Lewis SJ, Orland BI. The importance and impact of evidence based medicine. J Manag Care Pharm. 2004;10(5 Supp A):S3. doi:10.18553/jmcp.2004.10.S5-A.S3

9. Katrina A, Freeman EE, Robert D. The role of systematic reviews and meta-analysis in dermatology. J Invest Dermatol. 2012;132(11): e2.

10. Wu LL, Lin ZK, Weng HD, et al. Effectiveness of meditative movement on COPD: a systematic review and meta-analysis. Int $J$ Chron Obstruct Pulmon Dis. 2018;13:1239-1250. doi:10.2147/COPD. $\mathrm{S} 159042$

11. Ngai SP, Jones AY, Tam WW. Tai Chi for chronic obstructive pulmonary disease (COPD). Cochrane Database Syst Rev. 2016;6(6): CD009953.

12. Liu JP. Methodological quality assessment of clinical trials in traditional Chinese medicine: the principles of evidence-based medicine. Zhong Xi Yi Jie He Xue Bao. 2006;4(1):1.

13. Page MJ, Moher D. Evaluations of the uptake and impact of the Preferred Reporting Items for Systematic reviews and Meta-Analyses (PRISMA) statement and extensions: a scoping review. Syst Rev. 2017;6(1):263. doi:10.1186/s13643-017-0663-8

14. Page M, Shamseer L, Altman D, et al. Epidemiology and reporting characteristics of systematic reviews of biomedical research: a CrossSectional Study. PLoS Med. 2016;13(5):e1002028. doi:10.1371/journal.pmed. 1002028

15. Glasziou P, Altman DG, Bossuyt P, et al. Reducing waste from incomplete or unusable reports of biomedical research. Lancet. 2014;383(9913):267-276. doi:10.1016/S0140-6736(13)62228-X

16. David M, Alessandro L, Jennifer T, et al. Preferred reporting items for systematic reviews and meta-analyses: the PRISMA statement. Rev Española De Nutrición Hum Y Diet. 2009;18(3):e123.

17. Hartling L, Chisholm A, Thomson D, et al. A descriptive analysis of overviews of reviews published between 2000 and 2011. PLoS One. 2012;7(11):e49667. doi:10.1371/journal.pone.0049667

18. Shea BJ, Reeves BC, Wells G, et al. AMSTAR 2: a critical appraisal tool for systematic reviews that include randomised or non-randomised studies of healthcare interventions, or both. BMJ. 2017;358: j4008. doi:10.1136/bmj.j4008

19. Farquhar CM, Showell MG, Eae S, et al. Clinical trial registration was not an indicator for low risk of bias. $J$ Clin Epidemiol. 2017;84:47. doi:10.1016/j.jclinepi.2016.11.011

20. Moher D, Cook DJ, Eastwood S, et al. Improving the quality of reports of meta-analyses of randomised controlled trials: the QUOROM statement. Br J Surg. 2000;23(6):1448-1454.

21. Zorzela L, Loke YK, Ioannidis JP, et al. PRISMA harms checklist: improving harms reporting in systematic reviews. BMJ. 2016;352: i157. doi:10.1136/bmj.i157

22. Stewart LA, Mike C, Maroeska R, et al. Preferred reporting items for systematic review and meta-analyses of individual participant data: the PRISMA-IPD statement. J Am Med Assoc. 2015;313(16):16571665. doi:10.1001/jama.2015.3656

23. Schünemann H, Brożek J, Guyatt G, et al. The GRADE handbook. 2013. Available from: https://gdt.gradepro.org/app/handbook/hand book.html\#h.hnedbo8gqjqk. Accessed May 27, 2021.

24. Wei S, Huan Z, Wei C, et al. A meta-analysis of the effect of taijiquan exercise on pulmonary function rehabilitation in patients with stable chronic obstructive pulmonary disease. Chin J Rehabil Med. 2016.

25. Tian L, Li L, Zhang Y, et al. A meta-analysis of the effect of taijiquan on the rehabilitation of patients with stable chronic obstructive pulmonary disease. Chin J Gerontol. 2015.

26. Wang J, Liu X, Jun H, et al. Effects of Tai Chi exercise on lung function and exercise endurance of old patients with chronic obstructive pulmonary disease: a meta-analysis. Chin J Tissue Eng Res. 2015;5:815-820. 
27. Wang L, Gao L. Meta analysis of intervention effect of taijiquan on elderly patients with COPD. J Guangzhou Sport Univ. 2020;40 (6):95-101.

28. Wang C, Zhou L. Meta-analysis of the effects of taijiquan on lung function, exercise ability and quality of life in patients with chronic obstructive pulmonary disease. J Mod Med. 2020;36(22):3651-3656.

29. Dong J, Ji Y, Ge Y. Meta-analysis of Tai Chi exercise on rehabilitation of middle-aged and elderly patients with chronic obstructive pulmonary disease. J Jilin Sport Univ. 2021;37:1.

30. Guo JB, Chen BL, Lu YM, et al. Tai Chi for improving cardiopulmonary function and quality of life in patients with chronic obstructive pulmonary disease: a systematic review and meta-analysis. Clin Rehabil. 2016;30(8):750. doi:10.1177/0269215515604903

31. Wu W, Liu X, Wang L, et al. Effects of Tai Chi on exercise capacity and health-related quality of life in patients with chronic obstructive pulmonary disease: a systematic review and meta-analysis. Int $J$ Chron Obstruct Pulmon Dis. 2014;2014:1253-1263. doi:10.2147/ COPD.S70862

32. Jun-Hong Y, Yong-Zhong G, Hong-Mei Y, et al. Effects of Tai Chi in patients with chronic obstructive pulmonary disease: preliminary evidence. PLoS One. 2013;8(4):e61806. doi:10.1371/journal. pone.0061806

33. Guo C, Xiang G, Xie L, et al. Effects of Tai Chi training on the physical and mental health status in patients with chronic obstructive pulmonary disease: a systematic review and meta-analysis. J Thorac Dis. 2020;12(3):504-521. doi:10.21037/jtd.2020.01.03

34. Chen Y-W, Hunt MA, Campbell KL, et al. The effect of Tai Chi on four chronic conditions - cancer, osteoarthritis, heart failure and chronic obstructive pulmonary disease: a systematic review and meta-analyses. Br J Sports Med. 2016;50(7):397-407. doi:10.1136/ bjsports-2014-094388

35. Berry CE, Drummond MB, Han MK, et al. Relationship between lung function impairment and health-related quality of life in COPD and interstitial lung disease. Chest. 2012;142(3):704-711. doi:10.1378/chest.11-1332

36. Donohue JF. Minimal clinically important differences in COPD lung function. $J$ Chronic Obstr Pulm Dis. 2005;2(1):111-124. doi:10.1081/COPD-200053377

37. Lu M, Yao WZ. [Interpretation of Global Strategy for the Diagnosis, Management and Prevention of Chronic Obstructive Lung Disease (GOLD) 2016]. Zhonghua Yi Xue Za Zhi. 2016;96 (34):2689. Chinese.

38. Spruit MA, Watkins ML, Edwards LD, et al. Determinants of poor 6min walking distance in patients with COPD: the ECLIPSE cohort. Respir Med. 2010;104(6):849-857. doi:10.1016/j.rmed.2009.12.007

39. Crapo RO, Casaburi R, Coates AL, et al. ATS statement: guidelines for the six-minute walk test. Am J Respir Crit Care Med. 2002;166 (1):111-117.
40. Hernandes NA, Wouters EFM, Meijer K, et al. Reproducibility of 6minute walking test in patients with COPD. Eur Respir J. 2011;38 (2):261-267. doi:10.1183/09031936.00142010

41. Puhan MA, Mador MJ, Held U, et al. Interpretation of treatment changes in 6-minute walk distance in patients with COPD. Eur Respir J. 2009;5(2):56.

42. Weatherall M, Marsh S, Shirtcliffe P, et al. Quality of life measured by the St George's respiratory questionnaire and spirometry. Eur Respir J. 2009;33(5):1025-1030. doi:10.1183/09031936.00116808

43. Sanchez FF, Faganello MM, Tanni SE, et al. Relationship between disease severity and quality of life in patients with chronic obstructive pulmonary disease. Braz J Med Biol Res. 2008;41(10):860. doi:10.1590/S0100-879X2008005000043

44. Sideri S, Papageorgiou SN, Eliades T. Registration in the international prospective register of systematic reviews (PROSPERO) of systematic review protocols was associated with increased review quality. $J$ Clin Epidemiol. 2018;100:103-110. doi:10.1016/j. jclinepi.2018.01.003

45. Xu C, Cheng -L-L, Liu Y, et al. Protocol registration or development may benefit the design, conduct and reporting of dose-response metaanalysis: empirical evidence from a literature survey. BMC Med Res Methodol. 2019;19(1):78. doi:10.1186/s12874-019-0715-y

46. Ge L, Tian JH, Li YN, et al. Association between prospective registration and overall reporting and methodological quality of systematic reviews: a meta-epidemiological study. J Clin Epidemiol. 2018;93:45-55. doi:10.1016/j.jclinepi.2017.10.012

47. Jadad AR, Moore RA, Carroll D, et al. Assessing the quality of reports of randomized clinical trials: is blinding necessary? Control Clin Trials. 1996;17(1):1-12. doi:10.1016/0197-2456(95)00134-4

48. Koletsi D, Fleming PS, Michelaki I, et al. Heterogeneity in cochrane and non-cochrane meta-analyses in orthodontics. J Dent. 2018;74: S0300571218301106. doi:10.1016/j.jdent.2018.05.003

49. Tacconelli E. Systematic reviews: CRD's guidance for undertaking reviews in health care. Lancet Infect Dis. 2010;10(4):226. doi:10.1016/S1473-3099(10)70065-7

50. Higgins JPT, Thompson SG. Quantifying heterogeneity in a metaanalysis. Stat Med. 2002;21(11):1539-1558. doi:10.1002/sim.1186

51. Higgins J, Green S. Cochrane Handbook for Systematic Reviews of Interventions. Version 5.0.0 [Updated February 2008]. The Cochrane Collaboration; 2008.

52. Sterne JA, Sutton AJ, Ioannidis JP, et al. Recommendations for examining and interpreting funnel plot asymmetry in meta-analyses of randomised controlled trials. BMJ. 2011;343(jul22 1):d4002. doi:10.1136/bmj.d4002
International Journal of General Medicine

\section{Publish your work in this journal}

The International Journal of General Medicine is an international, peer-reviewed open-access journal that focuses on general and internal medicine, pathogenesis, epidemiology, diagnosis, monitoring and treatment protocols. The journal is characterized by the rapid reporting of reviews, original research and clinical studies

\section{Dovepress}

across all disease areas. The manuscript management system is completely online and includes a very quick and fair peer-review system, which is all easy to use. Visit http://www.dovepress.com/ testimonials.php to read real quotes from published authors. 